\title{
Transparencia online como bien intangible del sector público
}

\author{
Online transparency as an intangible \\ asset of the public sector
}

\author{
Carmen María ROBLES-LÓPEZ1 (iD) 0000-0003-4767-7169 \\ Rocío ZAMORA-MEDINA' (iD) 0000-0002-0541-2456
}

\section{Resumen}

Las organizaciones públicas se ven obligadas a afrontar la gestión de su intangibilidad de muy diversas formas. Por lo que respecta al bien intangible de la transparencia, la implantación de diferentes leyes específicas (a nivel nacional y regional) que afectan el cómo se presenta la información pública ha sido una práctica común en el caso de las administraciones públicas españolas. Este estudio analiza las consecuencias de la puesta en marcha de dichas leyes a partir de la evolución del nivel de transparencia que presentan las administraciones locales en sus websites, tomando como caso de estudio los ayuntamientos de la Región de Murcia durante tres oleadas consecutivas (2015, 2016 y 2017). Para ello, se utilizaron 52 indicadores (testados a nivel nacional) a partir de los cuales se obtuvieron interesantes resultados que demuestran que, cinco años después de la implantación de la ley, se sigue apreciando un déficit generalizado en la implementación real de la normativa de transparencia, que afecta cuestiones clave que repercuten sobre la intangibilidad. A la vez, se advierten diferencias significativas en el grado de cumplimiento en función del sexo de los alcaldes que están al frente del municipio.

Palabras clave: Comunicación. Gobierno local. Información pública. Participación ciudadana. Transparencia pública.

\begin{abstract}
Public organizations mustface the management of their intangible assets in different ways. Regarding transparency, the implementation of different specific laws (local and nationwide) which affect the way public information is presented has been a common practice in the case of Spanish public administrations. This study analyses the consequences of the application of such laws by assessing the evolution of the level of transparency presented by local administrations, taking as a case study all Councils in the Region of Murcia. Data collected in three waves $(2015,2016$, and 2017) have been included in order to measure the evolution of this intangible asset in a timeline. For the analysis of the online transparency level, 52 indicators were used (tested nationwide). The results show that, five years after the implementation of the law, even though there has been a slight improvement, a generalized deficit in the real implementation of the transparency regulation is still visible, which affects key points related to its intangibility. At the same time, there are significant differences in the degree of compliance depending on the sex of the mayors who were in charge of the municipality.
\end{abstract}

Keywords: Communication. Local government. Public information. Civic participation. Public transparency.

\footnotetext{
1 Universidad de Murcia, Facultad de Comunicación y Documentación, Departamento de Información y Documentación. Campus Universitario Espinardo, 30100, Murcia, España. Correspondencia para/Correspondence to: R. ZAMORA-MEDINA E-mail: <rzamoramedina@um.es>.

Apoyo: Ministerio de Educación y Ciencia de España (Ref: CSO 2013-46997-R).

Recibido el 2 de julio de 2019, presentado el 24 de enero de 2020 y aprobado el 6 de marzo de 2020.
}

Cómo citar este artículo/How to cite this article

Robles-López, C. M.; Zamora-Medina, R. Transparencia online como bien intangible del sector público. Transinformação, v. 32, e190059, 2020. http:// dx.doi.org/10.1590/1678-9865202032e190059 


\section{Introducción}

El interés por estudiar el fenómeno global de la transparencia, que afecta a todo tipo de instituciones y organizaciones, se ha visto acelerado en parte debido a factores entre los que se encuentran la pérdida de legitimidad de los sistemas políticos, económicos y sociales, derivada principalmente de la crisis económica y de la mala gestión de los recursos; el aumento de la cultura democrática en los países y la menor permisividad de la corrupción; así como por el desarrollo de leyes sobre acceso a la información pública en altos rangos normativos (López-López; Vaca-Tapia; Rodríguez-Navas, 2017)

A raíz de la aprobación de la Ley 19/2013 Española de Transparencia, Acceso a la Información Pública y Buena Gobernanza (España, 2013), las instituciones públicas sienten la necesidad de mejorar la forma en la que se comunican, en aras a reconstruir la confianza en el sector público por parte de los ciudadanos. Tal y como ha demostrado la literatura existente en relación con este objeto de estudio, en el ámbito de la información pública online, esta apuesta por la transparencia resulta esencial, especialmente en los contextos locales, donde la relación entre los ciudadanos y las instituciones es más cercana (Kim; Lee, 2012; Beltrán-Orenes; Martínez Pastor, 2016; Rebolledo; Zamora-Medina; Rodríguez-Virgili, 2016).

En esta línea, surge la necesidad de gestionar mejor los bienes intangibles en el sector público para dar respuesta a las exigencias sociales de transparencia y excelencia en los servicios públicos (Bossi; Fuentes; Serrano, 2005; MacNamara, 2018). Tomando como referencia el marco conceptual más destacado sobre este objeto de estudio (Canel, 2017a; 2017b; 2018), este trabajo considera como bien intangible a aquellos bienes no monetarios que carecen de sustancia física y que dan acceso al capital tangible. Dichos bienes están construidos con eventos pasados de la organización o, lo que es lo mismo, están unidos al comportamiento de la organización y proporcionan un recurso que lleva asociado un beneficio económico a largo plazo como, por ejemplo, la supervivencia de la organización.

Son muchos los autores que consideran que la intangibilidad en el sector público es mayor que la del sector privado (Cinca; Molinero; Bossi, 2003; Bossi; Fuentes; Serrano, 2005; Queiroz; Callén; Cinca, 2005). El sector público y su relación con la intangibilidad, por tanto, ha permanecido como un territorio que requiere de mayor exploración (Luoma-aho; Makikangas, 2014) así como de un mayor control interno (Poveda; Tituaña; Franco, 2016).

Con estas circunstancias, a la Administración Pública se le presenta el reto de recuperar la confianza de los ciudadanos (Canel, 2018), tal como han recogido diferentes informes de organismos internacionales (Organización de Cooperación y Desarrollo Económicos; informes de Naciones Unidas, el Banco Mundial, la Fundación Internacional Monetaria, la Organización de Estados Unidos y el Banco de Desarrollo de América Latina) y en investigaciones recientes (Canel, 2017a; 2017b; Robles, 2017). Estos organismos ponen de manifiesto la necesidad de investigar sobre la recuperación de la confianza en las instituciones públicas. Por medio de estos informes, se ha hecho una llamada al sector público para trabajar en aspectos tales como mejorar la transparencia y la responsabilidad, el acceso a la información, etc. De esta forma se pretende acercar las instituciones públicas a los ciudadanos (Robles; Rodríguez, 2017).

En concreto, la mayoría de la literatura sobre transparencia en el sector público se ha centrado sobre quién toma y cómo se toman las decisiones, pero no tanto en la relación entre la transparencia y los ciudadanos (Rubiños, 2017).

En esta investigación, nos vamos a basar en la definición que hacen Stirton y Lodge (2001) sobre el concepto de transparencia. Según Rubiños (2017, p. 173), estos autores consideran que:

Las organizaciones transparentes son aquellas que promueven la visibilidad del proceso de toma de decisiones, están abiertas a las aportaciones de los ciudadanos, ofrecen a sus públicos la mayor cantidad de opciones posibles y cooperan con otras organizaciones para alcanzar objetivos comunes que beneficien a todos. 
Tras esta definición, observamos que la transparencia no cumple con las características de un bien intangible. Sin embargo, si se tienen en cuenta otros factores, no solo la apertura de información puede dar lugar a otros bienes intangibles tales como la confianza y la legitimidad. De forma que, proporciona valor a largo plazo a la institución pública y a los ciudadanos (Rubiños, 2017).

Actualmente, la transparencia es concebida como una respuesta por parte de la organización pública a la demanda de un gobierno más abierto (Rebolledo; Zamora-Medina; Rodríguez-Virgili, 2017). Por lo que respecta a los ciudadanos, la transparencia supone también una forma de participar en las actividades públicas, en la toma de decisiones y en la gestión de asuntos públicos; es un modo de reducir la corrupción y reforzar la participación (Stasavage, 2003).

Con todo, las organizaciones y sus representantes políticos tienen ante sí el reto de afrontar una situación de desconfianza debido a la corrupción y a la crisis económica con una apuesta clara por la adecuada gestión de su comunicación pública (Pasquier; Villeneuve, 2017). Bajo estas condiciones, la exigencia de los ciudadanos hacia sus representantes políticos incluye además el requisito de que, tras su elección, estos deban rendir cuentas de sus responsabilidades (Bauhr; Grimes, 2014). Por tanto, la administración pública tiene que ofrecer nuevas formas con las que generar la implicación ciudadana en la toma de decisiones para lograr gobernar con los ciudadanos (Canel, 2018). Desde esta presunción, los procesos electorales puntuales ya no son suficientes como forma de participación ciudadana en la democracia (Verge, 2007). Es necesario un cambio de mentalidad en la transparencia y en la participación cuando se gestiona información pública (Rebolledo; Zamora-Medina; Rodríguez-Virgili, 2017).

En definitiva, resulta esencial que la administración preste atención a la adecuada gestión de la transparencia que afecta a la información pública que ofrece a la sociedad, considerando que será sin duda un elemento esencial para recuperar la confianza ciudadana y la credibilidad en el sector público. En este sentido, la transparencia no debe ser considerada una simple obligación. Representa más bien una oportunidad para que la organización pueda construir "una relación basada en el beneficio mutuo". De este modo, "la organización obtendría legitimidad, generaría confianza y construiría una buena reputación", que sin duda aumentará el valor de otros bienes intangibles (Rubiños, 2017, p. 176).

\section{La transparencia como bien intangible del sector público}

La transparencia, como objeto de estudio, se ha convertido en un tema recurrente en la investigación académica en áreas como la Comunicación Política y la Ciencia Política (García, 2014). En un momento marcado por la pérdida de credibilidad de los ciudadanos hacia la política, una comunicación basada en la transparencia se presenta como un aspecto esencial para recuperar la confianza de los ciudadanos en sus representantes políticos. Tal y como advierten diversos académicos, la transparencia se liga a la calidad democrática de un país (Alonso-Muñoz; Casero-Ripollés, 2017), siendo su poco nivel sinónimo de precariedad (Manfredi-Sánchez, 2017) y un exceso como un mecanismo de pérdida de privacidad (Han, 2013), de autonomía personal y de gestión.

Consideremos la definición contenida en la Guía de lenguaje claro de la Organización No Gubernamental Transparencia Internacional (Transparency, 2009, p. 48), que entiende por transparencia:

La cualidad de un gobierno, empresa, organización o persona que debe ser abierta en la divulgación de información, políticas, planes, procesos y acciones. Como regla general, los empleados públicos, gerentes y directores de compañías y organizaciones, y los consejos tienen la obligación de actuar de manera visible, previsible y comprensible para promover la participación y la rendición de cuentas. 
En el marco de la llamada "democracia electrónica" o "ciberdemocracia", la transparencia como bien intangible es una condición esencial para que las organizaciones públicas puedan responder a la creciente demanda de una forma de gobierno más abierta. Pero más allá de la cuestión del acceso a la información pública, este término también está directamente relacionado con el control político y la rendición de cuentas, así como con la participación ciudadana.

Concretamente, la cultura de transparencia requiere, por un lado, un cambio de mentalidad en el uso de la información pública, con mayor ecuanimidad y pluralidad. No tiene sentido tener una enorme cantidad de documentos almacenados si no existe una política que los haga accesibles, de modo que fomente la consulta y promueva la interacción con los ciudadanos y los políticos, asumiendo también el deber de rendir cuentas por sus responsabilidades (Bauhr; Grimes, 2014; Schiavi, 2017).

Por lo que respecta a la rendición de cuentas, la transparencia refleja el deseo de la ciudadanía de conocer lo que sucede dentro de las instituciones públicas, condición esencial para el funcionamiento democrático de la política. La transparencia se ha convertido, de este modo, en un valor fundamental de la democracia (Uvalle-Berrones, 2016), al facilitar el monitoreo de la actividad de los cargos públicos y dificultar, así, los abusos de poder que proliferan en contextos de opacidad informativa. En este sentido, cuanto más perciban los ciudadanos que tienen a su disposición la información necesaria para ejercer un control efectivo de la gestión de los asuntos públicos, más se reducirá la brecha existente entre los gobernantes y gobernados, un hecho sobre el que se sustenta la crisis de la democracia representativa.

Hoy en día, los representantes públicos y los partidos políticos también se enfrentan a un escenario complicado, especialmente en relación con la participación ciudadana, aspecto cada vez más exigido por parte de la sociedad civil. Las elecciones ya no representan la forma más generalizada en la que los ciudadanos participan en la democracia, sino que ahora están surgiendo otras acciones, favorecidas por las nuevas tecnologías y relacionadas con la regeneración democrática (Verge, 2007). En este contexto, la transparencia de las actividades públicas es, a su vez, una condición clave para fomentar la participación ciudadana en la toma de decisiones y en la gestión de los asuntos relevantes, así como para reducir las injusticias e, idealmente, la corrupción (Stasavage, 2003). De esta manera, la demanda de transparencia es un reclamo básico para avanzar hacia formas más participativas de democracia.

En España, existen diferentes estudios previos que ofrecen una reflexión sobre la transparencia y su relación con la responsabilidad de los gobiernos (Alguacil-Gómez, 2006; Guerrero, 2006; Labio Bernal, 2006; MolinaRodríguez-Navas, 2008; Reig, 2009; Villoria-Mendieta, 2015; Gértrudix; Gertrudis-Casado; Álvarez-García, 2016). Pero ha sido a raíz de la entrada en vigor a nivel local, en diciembre de 2015, de la Ley 19/2013 de Transparencia, Acceso a la Información Pública y Buen Gobierno (España, 2013), cuando han proliferado el mayor número de trabajos realizados en España sobre este objeto de estudio. En concreto, esta ley obliga a las administraciones públicas a publicar activamente la información institucional relevante en sus sitios web y a responder a las solicitudes de información pública de los ciudadanos (Beltrán-Orenes; Martínez Pastor, 2016; Manfredi-Sánchez et al., 2016).

Esta investigación forma parte del marco del proyecto titulado "Comunicación y periodismo para la participación ciudadana en el monitoreo y evaluación de la Administración del gobierno local" (Ciencias Sociales 2012-34687) sobre el que se han publicado diferentes resultados y conclusiones sobre el tema (Moreno-Sardà; Molina-Rodríguez-Navas; Simelio-Solà, 2017; Rebolledo; Zamora-Medina; Rodríguez-Virgili, 2016; Manfredi-Sánchez, 2017). Se ha elegido el contexto local como escenario para esta investigación, precisamente porque consideramos que es en la administración local donde la necesidad de transparencia, responsabilidad y participación quizá sea más clara y evidente (Villoria-Mendieta, 2015). De hecho, la gestión de la comunicación local representa el entorno más cercano en el que se resuelven la mayoría de los asuntos de la vida cotidiana, en contraste con los problemas nacionales y globales (Zamora-Medina, 2011). También las administraciones locales, por su cercanía, ofrecen un 
mayor escenario de confianza para los ciudadanos, en comparación con las instituciones nacionales (Kim; Lee, 2012).

Diferentes estudios previos han reconocido el nivel insatisfactorio de la transparencia en los sitios web de los ayuntamientos en España, revelando deficiencias en la rendición de cuentas lideradas por los gobiernos (Cabezuelo-Lorenzo; Rey-García; Tapia-Frade, 2016; Manfredi-Sánchez et al., 2016; Rebolledo; Zamora-Medina; Rodríguez-Virgili, 2016, Alcaraz-Quiles; Navarro-Galera; Ortiz-Rodríguez, 2017; Rebolledo; Zamora-Medina; Rodríguez-Virgili, 2017). Estos trabajos previos no solo señalan que la cantidad de información pública disponible en las plataformas es deficiente sino que, además, la manera en la que se proporciona a los ciudadanos dicha información resulta inadecuada: falta información relevante (proyectos legislativos, acuerdos y contratos firmados, planes de acción); los datos que aparecen en los sitios web no están bien explicados y transmitidos a los ciudadanos; la información presentada no es neutral, accesible y confiable, etc.).

Partiendo de estos trabajos previos, nuestro objetivo es, por un lado, comprobar el grado de cumplimiento de dichos indicadores en su evolución en el tiempo, con el fin de comprobar si ha existido una tendencia a la mejora o no con el transcurso del tiempo. Por otro lado, también nos proponemos testar si el hecho de que exista una mayor presencia de mujeres alcaldesas, como nota característica de la Región de Murcia (en comparación con el resto de las administraciones locales de España), ha podido marcar alguna diferencia con respecto al cumplimiento de los indicadores de transparencia. Estos objetivos se concretan en las siguientes hipótesis: Existen diferencias significativas en lo que se refiere al cumplimiento de los indicadores de transparencia en función del tiempo transcurrido. De tal forma que, conforme pasa el tiempo, el grado de cumplimiento de todos los indicadores es mayor y existen diferencias significativas en lo que se refiere al cumplimiento de los indicadores de transparencia en función del sexo del alcalde del municipio.

\section{Herramientas y Procedimientos}

El estudio que aquí se presenta pretende evaluar la evolución del nivel de transparencia que presentan las administraciones locales, tomando como caso de estudio los ayuntamientos de los 45 municipios de la Región de Murcia. En concreto, se incluyen datos del seguimiento realizado durante tres oleadas (2015, 2016 y 2017), con el fin de poder medir la evolución de este bien intangible en el tiempo.

Para el análisis del nivel de transparencia online se utilizaron 52 indicadores a partir de los cuales fueron evaluadas las webs municipales en los tres momentos que conforman la muestra del análisis. Aclaramos que, en el primer análisis realizado, el número de indicadores para testar el nivel de transparencia en los municipios era algo inferior. En concreto, se utilizaron 41 indicadores basados en el decálogo de las buenas prácticas de la comunicación pública local. En la segunda y tercera ola, sin embargo, se incrementaron los indicadores hasta llegar a los 52, con el objetivo de adaptar el análisis a la nueva ley de transparencia española. Por tanto, en esta investigación se incluye un análisis cuantitativo sobre la evolución de la presencia o ausencia de los indicadores empleados para medir el nivel de transparencia y participación en las páginas web de los ayuntamientos de la Región de Murcia.

Por otro lado, es importante señalar que los indicadores empleados están sustentados y testados a nivel nacional en el Mapa Infoparticipa (Moreno-Sardá et al., 2013). Con dichos indicadores hemos podido evaluar la información de las páginas web, que está relacionada con las siguientes cuestiones: quiénes son los representantes políticos; cómo se manejan los recursos comunes; cómo se proporciona información sobre su gestión; y qué herramientas se publican para facilitar la participación pública.

En este sentido, podemos afirmar que los indicadores que se han empleado en las tres olas del análisis han sido diseñados para valorar la disposición de la información básica en las páginas web de los ayuntamientos. Durante el análisis de las páginas web hemos tenido en cuenta algunas consideraciones: en primer lugar, hemos 
identificado la página web que corresponde a cada ayuntamiento, teniendo en cuenta que a veces no tiene el mismo nombre que el municipio; en segundo lugar, como la información que hemos valorado se considera básica, hemos considerado que se evalúa aquella que está disponible en menos de tres clics; en tercer lugar, hemos tenido en cuenta que las páginas web estuviesen actualizadas. Si no se sigue un proceso de actualizaciones, la información se queda obsoleta y tenemos el efecto contrario.

En cuanto al procesamiento que hemos llevado a cabo para el análisis, en un primer lugar, hemos recogido todos los datos en una hoja de cálculo de Excel y después estos datos han sido analizados con el programa estadístico SPSS ${ }^{\circledR}$ versión 21 (International ..., 2012). Una vez recopilados todos los datos, hemos realizado un análisis comparativo de la información extraída de todas las páginas web de los ayuntamientos de la Región de Murcia.

\section{Resultados}

Para la exposición de resultados se sigue el orden de las preguntas e hipótesis de investigación. En primer lugar, recogeremos los datos básicos obtenidos en los tres años analizados, diferenciando el sexo del alcalde o alcaldesa; en segundo lugar, el cumplimiento de los indicadores analizados durante el periodo analizado; y, por último, el cumplimiento de los indicadores analizados distinguiendo por sexo del alcalde o alcaldesa.

La mayoría de los municipios incluidos en la muestra de análisis son pequeños (62,2\%), es decir, con menos de 10.000 habitantes, mientras que apenas un tercio de los mismos pueden ser considerados municipios medianos (33,3\%), con entre 10.001 y 100.000 habitantes. Los municipios con más de 100.000 habitantes, considerados los municipios de gran tamaño, apenas alcanzan el 4,4\% de la muestra total.

Un dato interesante a tener en cuenta es que, a lo largo de los años, en los ayuntamientos de la Región de Murcia se ha ido incrementando el número de alcaldesas frente al número de alcaldes. Así, mientras que en 2015 solo había un 20,0\% de mujeres, en 2017 ese porcentaje ya alcanzaba el 35,6\%.

El análisis exhaustivo que hemos realizado de los datos nos permite conocer el porcentaje de cumplimiento de cada uno de los indicadores de transparencia por parte de los ayuntamientos de la Región de Murcia. A continuación, se ofrecen los resultados distribuidos en bloques de indicadores: un primer bloque dedicado a la transparencia de la organización y otro dedicado a la información orientada a la participación ciudadana. Los resultados se ofrecen de forma comparativa entre los estudios realizados en 2015, 2016 y 2017.

En esta investigación hemos puntuado positivamente un indicador cuando en la página web encontramos la información sobre la cuestión planteada. O, también, si las herramientas de participación corresponden con la utilidad que se establece. Asimismo, hemos considerado que, tanto las informaciones como las herramientas, tienen que ser de fácil acceso. Es decir, una persona que no es experta tiene que poder acceder con un máximo de tres clics.

\section{Transparencia de la Organización}

Indicadores sobre quiénes son los representantes políticos

En esta primera categoría de indicadores que encontramos en la Tabla 1, los ayuntamientos analizados en la primera oleada solo aprobaban con un 82,2\% el indicador de información básica sobre el alcalde o alcaldesa. Este indicador ha ido incrementando progresivamente en 2016 (86,7\%) y en 2017 (93,3\%). En algunos casos, la información estaba separada por varias secciones de la página web. 
Tabla 1. Porcentaje de cumplimiento de los indicadores del nivel de transparencia sobre quiénes son los representantes políticos. Región de Murcia, España, 2019.

\begin{tabular}{|c|c|c|c|c|c|c|}
\hline \multirow{2}{*}{ Indicador } & \multicolumn{2}{|c|}{2015} & \multicolumn{2}{|c|}{2016} & \multicolumn{2}{|c|}{2017} \\
\hline & $\mathrm{n}$ & $\%$ & $\mathrm{n}$ & $\%$ & $n$ & $\%$ \\
\hline $\begin{array}{l}\text { ¿Se da información básica sobre el alcalde o la alcaldesa: nombre y } \\
\text { apellidos, foto y partido político? }\end{array}$ & 37 & 82,2 & 39 & 86,7 & 42 & 93,3 \\
\hline $\begin{array}{l}\text { ¿Se da información básica sobre el alcalde o la alcaldesa: biografía y/o } \\
\text { currículum? }\end{array}$ & 9 & 20,0 & 23 & 51,1 & 28 & 62,2 \\
\hline $\begin{array}{l}\text { ¿Se da información básica sobre los representantes que forman parte } \\
\text { del gobierno: nombre y apellidos, foto y partido político? }\end{array}$ & 18 & 40,0 & 31 & 68,9 & 31 & 68,9 \\
\hline $\begin{array}{l}\text { ¿Se da información básica sobre los representantes que forman parte } \\
\text { del gobierno: biografía y/o currículum? }\end{array}$ & 5 & 11,1 & 18 & 40 & 23 & 51,1 \\
\hline $\begin{array}{l}\text { ¿Se da información básica sobre los representantes que no forman } \\
\text { parte del gobierno: nombre y apellidos, foto y partido político? }\end{array}$ & 11 & 24,4 & 14 & 31,1 & 25 & 55,6 \\
\hline $\begin{array}{l}\text { ¿Se da información básica sobre los representantes que no forman } \\
\text { parte del gobierno: biografía y/o currículum? }\end{array}$ & 3 & 6,7 & 7 & 15,6 & 15 & 33,3 \\
\hline $\begin{array}{l}\text { ¿Se publican las retribuciones (mensuales y/o anuales) de los } \\
\text { representantes políticos? }\end{array}$ & & - & 20 & 44,4 & 22 & 48,9 \\
\hline $\begin{array}{l}\text { ¿Se publican las declaraciones de actividades y bienes de los cargos } \\
\text { electos? }\end{array}$ & & - & 14 & 31,1 & 16 & 35,6 \\
\hline ¿Se publica la agenda institucional del alcalde? & & & 21 & 46,7 & 24 & 53,3 \\
\hline $\begin{array}{l}\text { ¿Se publican en la web datos de contacto de los miembros del } \\
\text { gobierno (correo electrónico y/o Twitter y/o Facebook y/o página web } \\
\text { y/o teléfono...)? }\end{array}$ & & 20,0 & 26 & 57,8 & 30 & 66,7 \\
\hline $\begin{array}{l}\text { ¿Se publican en la web datos de contacto de los miembros de la } \\
\text { oposición (correo electrónico y/o Twitter y/o Facebook, y/o página } \\
\text { web, y/o teléfono? }\end{array}$ & & 4,4 & 8 & 17,8 & 13 & 28,9 \\
\hline
\end{tabular}

Fuente: Elaborada por los autores, 2019.

Por otro lado, es importante señalar que cada vez más ayuntamientos han incluido datos biográficos y de currículum del alcalde o alcaldesa. Del año 2015 al año 2016 se ha duplicado y en 2017 hay un pequeño incremento respecto al año anterior.

En cuanto a la información básica que se ofrece sobre los representantes políticos municipales, sigue siendo poco usual. Los datos reflejan que el porcentaje de este indicador ha subido ligeramente de la primera a la segunda oleada, pero no en la tercera. Sin embargo, sí podemos observar que ha incrementado la información sobre la biografía y el currículum de los representantes políticos municipales de un 11,1\% en 2015 a un 51,1\% en 2017.

Sin embargo, respecto a la oposición, los porcentajes de cumplimientos son bastante menores, aunque se observa una ligera mejoría entre las tres oleadas (apenas alcanzan al 50,0\% en el caso de la tercera oleada). En la primera y segunda oleada casi no se proporciona información sobre la biografía y el currículum de la oposición, el $6,7 \%$ y el 15,6\% respectivamente. En la tercera, el indicador sube a un 33,3\%. Por tanto, este dato aun siendo bajo, se ha duplicado de una oleada a otra.

De los nuevos indicadores incluidos en la segunda y tercera oleada, los datos muestran que menos de un $50 \%$ de los alcaldes y alcaldesas publican sus retribuciones mensuales o anuales. Y, por otra parte, menos del 40\% publican sus declaraciones de bienes y actividades. Esta cuestión sigue suscitando, al parecer, demasiados recelos entre los representantes políticos locales.

También es importante destacar que la publicación de la agenda del alcalde y la alcaldesa sigue siendo una asignatura pendiente para más del 50\% de los ayuntamientos. Es un dato que demuestra cómo, a nivel local, aunque exista dicha agenda, no se considera que pueda ser de interés del ciudadano. 
Al medir los indicadores relativos a la información que ofrecen los representantes políticos considerando diferencias del sexo de los alcaldes o alcaldesas, se encontraron únicamente tres variables significativas, que se dan en diferente momento, por lo que no se puede considerar una tendencia clara. Los tres indicadores apuntan a un mayor grado de cumplimiento de los alcaldes que de las alcaldesas, en tales casos concretos. Por un lado, la que se refiere a la información básica sobre el alcalde/esa (nombre, apellido, foto y partido político), que en un 64,0\% de los casos en los que los ediles eran hombres se cumplía este indicador frente al 35,7\% de los casos en los que eran mujeres. Por otra parte, en cuanto a la información básica de los representantes que afecta a su biografía y currículum, los alcaldes cumplían en un 80,0\% y las alcaldesas, un 20,0\%. Finalmente, en cuanto a la publicación de actividades y bienes de los cargos electos, los alcaldes también puntuaron más alto $(71,4 \%)$ que las alcaldesas $(28,6 \%)$.

\section{Indicadores sobre cómo gestionan los recursos colectivos}

En este epígrafe vamos a analizar los indicadores sobre cómo se gestionan los recursos colectivos, recogidos en la Tabla 2. Los datos muestran que los porcentajes no son mucho mejores que en la tabla anterior. Los ayuntamientos de la Región de Murcia solo aprueban en las tres oleadas en dos indicadores: por un lado, cumplen al publicar las Ordenanzas Municipales y, por otro lado, también cumplen al ofrecer información sobre el Plan de Ordenación Urbanística u otras normas de planificación urbanística. Los datos en estos indicadores se asemejan en las tres oleadas.

Sin embargo, el indicador con mayor dificultad de cumplimiento, en las tres oleadas, resultó ser la información sobre el plan de gobierno, plan de actuación municipal y/o plan estratégico. De nuevo se observa cierta falta de planificación a nivel local que, o bien es inexistente, o bien no se hacen públicos como debieran.

El resto de los indicadores incluidos en este grupo son también bastante deficientes y no alcanzan la aprobación mayoritaria. Así, en cuanto a la información relativa a los órganos de gobierno municipales, menos del 32\% informa sobre el organigrama de los responsables y sus funciones (2016 y 2017).

Tabla 2. Porcentaje de cumplimiento de los indicadores del nivel de transparencia sobre cómo gestionan los recursos colectivos. Región de Murcia, España, 2019.

\begin{tabular}{|c|c|c|c|c|c|c|}
\hline \multirow{2}{*}{ Indicador } & \multicolumn{2}{|c|}{2015} & \multicolumn{2}{|c|}{2016} & \multicolumn{2}{|c|}{2017} \\
\hline & $n$ & $\%$ & $n$ & $\%$ & $n$ & $\%$ \\
\hline $\begin{array}{l}\text { ¿Se da información sobre la composición de los órganos de gobierno: pleno, } \\
\text { junta de gobierno y/o comisiones informativas? }\end{array}$ & 16 & 48,9 & 27 & 60 & 31 & 68,9 \\
\hline \multirow{2}{*}{$\begin{array}{l}\text { ¿Se da información sobre las competencias y el calendario de trabajo de estos } \\
\text { órganos de gobierno? }\end{array}$} & 16 & 35,6 & \multirow{2}{*}{18} & \multirow{2}{*}{40} & \multirow{2}{*}{13} & \multirow{2}{*}{28,9} \\
\hline & 30 & 66,7 & & & & \\
\hline $\begin{array}{l}\text { ¿Se publica el organigrama completo con los nombres y apellidos de las personas } \\
\text { responsables y sus funciones? }\end{array}$ & - & & 13 & 28,9 & 14 & 31,1 \\
\hline $\begin{array}{l}\text { ¿Se publican las convocatorias de los plenos municipales con los órdenes del día } \\
\text { previos a la celebración? }\end{array}$ & 11 & 24,4 & 24 & 53,3 & 22 & 48,9 \\
\hline ¿Se publican las actas del Pleno Municipal? & 14 & 31,1 & 25 & 55,6 & 22 & 48,9 \\
\hline $\begin{array}{l}\text { ¿Se publican los acuerdos completos de la Junta de Gobierno y/o las actas } \\
\text { íntegras, cuando la Junta de Gobierno actúe en delegación del pleno? }\end{array}$ & 5 & 11,1 & 16 & 35,6 & 16 & 35,6 \\
\hline $\begin{array}{l}\text { ¿Se da información sobre el Plan de Gobierno (PG), Plan de Actuación Municipal } \\
\text { (PAM) y/o Plan Estratégico? }\end{array}$ & 0 & 0 & 2 & 4,4 & 7 & 15,6 \\
\hline $\begin{array}{l}\text { ¿Se da información sobre el Plan de Ordenación Urbanística Municipal, Plan } \\
\text { General de Ordenación u otras normas de planificación urbanística, así como sus } \\
\text { modificaciones puntuales? }\end{array}$ & 29 & 64,4 & 30 & 66,7 & 34 & 75,6 \\
\hline ¿Se publican las ordenanzas municipales? & 33 & 73,3 & 39 & 86,7 & 38 & 84,4 \\
\hline
\end{tabular}

Fuente: Elaborada por los autores, 2019. 
Destaca un pequeño incremento de 2015 a 2016 en información que puede ser de interés para los ciudadanos como, por ejemplo, que se haga público el orden del día de los Plenos municipales, de las actas del Pleno Municipal y de los acuerdos de la Junta de Gobierno. Sin embargo, desciende ligeramente en 2017 (no más del 50\%). Se puede observar que no es una práctica que realicen la mayoría de los ayuntamientos.

Se detecta, asimismo, una cierta tendencia a mejorar el nivel de transparencia respecto a la información sobre la composición de los órganos de gobierno entre las tres oleadas del estudio, hasta alcanzar el 68,9\% en 2017. Sin embargo, resulta bastante deficitaria la información que se ofrece sobre las competencias y el calendario de trabajo de estos órganos de gobierno (un dato que en 2015 se recogió a partir de dos indicadores diferentes), que en ningún caso supera el 50,0\%.

Al contemplar este grupo de indicadores en función del sexo de los alcaldes o alcaldesas, encontramos diferencias significativas en 2015 y en 2016. En el primer caso, se llegaron a identificar hasta cuatro indicadores en los que el hecho de que el edil fuera hombre significó un porcentaje mucho más alto (sobre el 80,0\%) que para el caso de que fuera mujer. Se trata de los indicadores relativos a la publicación de la convocatoria de los plenos municipales, la publicación de los acuerdos de la Junta de Gobierno y las actas municipales, la información sobre el plan de gobierno, el plan de actuación municipal y el plan estratégico, así como la información sobre el plan de ordenación urbanística municipal y otras normas similares. En 2016 se identificó otra relación significativa entre el sexo de los alcaldes y la información sobre la composición de los órganos de gobierno, que apuntaba a resultados similares (70,4\% mayor cumplimiento en el caso de los alcaldes).

Indicadores sobre cómo se gestionan los recursos económicos: presupuestos, salarios, contrataciones, subvenciones, etc.

Como hemos visto anteriormente, la transparencia en la gestión municipal exige que los ciudadanos puedan acceder a una información objetiva, clara y detallada, es decir, que sea una información comprensible sobre las actuaciones, tanto del gobierno como de la oposición, incluyendo la gestión de los recursos públicos y las contrataciones. Los datos aquí incluidos apuntan a que los ayuntamientos de la Región de Murcia no facilitan de forma adecuada toda esta información a la que los ciudadanos tienen derecho, como podemos observar en la Tabla 3.

En la segunda oleada encontramos una mejora en el indicador básico que exige la publicación de los presupuestos municipales (62,2\%). Sin embargo, ha descendido en 2017 (57,8\%). Se considera un porcentaje muy bajo para ser una información tan importante desde el punto de vista económico. Tampoco la información más detallada sobre estos presupuestos, tales como la ejecución del presupuesto anual, se suele publicar mayoritariamente (este dato se incrementó de 2015 a 2016 y luego se mantuvo en 2017 en torno al 29,0\%) ni la información sobre el cumplimiento de objetivos de estabilidad presupuestaria o sostenibilidad financiera (no llegó al 32,0\% en 2017). Respecto a los demás indicadores, otra cuestión que merece una reflexión tiene que ver con el hecho de que los ayuntamientos deben dar mayor consideración a la información relativa a retribuciones de personal, listados y retribuciones de los directivos y cargos de confianza, ya que apenas se cumple este indicador en los ayuntamientos (en 2017 se incrementó mínimamente pero no alcanzaba el 25,0\%). Sin embargo, vemos que la información relativa a licitaciones en curso, ofertas de trabajo en los ayuntamientos, los procesos de concursos públicos y resultados sí que alcanzan niveles de transparencia más aceptables, superando el 70,0\% en 2017.

Con relación a las diferencias que resultaron significativas entre este grupo de indicadores y la variable sexo de los alcaldes, se detectó como significativa la misma variable en 2015 y 2016 relativa a la publicación del presupuesto del ayuntamiento del año en curso, que fue considerablemente mayor en el caso de los alcaldes que en el de las alcaldesas (rozando el 81\% y el 71\% en cada año). Asimismo, en 2015 también se detectó un mayor cumplimiento de los alcaldes en cuanto a la publicación del coste y las características de la campaña de publicidad institucional en medios de comunicación. 
Tabla 3. Porcentaje de cumplimiento de los indicadores del nivel de transparencia sobre cómo se gestionan los recursos económicos. Región de Murcia, España, 2019.

\begin{tabular}{|c|c|c|c|c|c|c|}
\hline \multirow{2}{*}{ Indicador } & \multicolumn{2}{|c|}{2015} & \multicolumn{2}{|c|}{2016} & \multicolumn{2}{|c|}{2017} \\
\hline & $\mathrm{n}$ & $\%$ & $n$ & $\%$ & $\mathrm{n}$ & $\%$ \\
\hline $\begin{array}{l}\text { ¿Se publica el Presupuesto del Ayuntamiento del año en curso y de } \\
\text { los organismos autónomos y entes dependientes, en caso de existir? }\end{array}$ & 21 & 46,7 & 28 & 62,2 & 26 & 57,8 \\
\hline $\begin{array}{l}\text { ¿Se publica información sobre la ejecución trimestral del presupuesto } \\
\text { del año en curso? }\end{array}$ & 4 & 8,9 & 13 & 28,9 & 13 & 28,9 \\
\hline ¿Se publican las modificaciones presupuestarias realizadas? & & - & 10 & 22,2 & 14 & 31,1 \\
\hline $\begin{array}{l}\text { ¿Se publica información sobre el cumplimiento de los objetivos de } \\
\text { estabilidad presupuestaria y sostenibilidad financiera y/o nivel de } \\
\text { endeudamiento? }\end{array}$ & - & - & 14 & 31,1 & 14 & 31,1 \\
\hline $\begin{array}{l}\text { ¿Se publica la liquidación de los presupuestos de ejercicios anteriores } \\
\text { y/o la cuenta general? }\end{array}$ & - & - & 20 & 44,4 & 23 & 51,1 \\
\hline $\begin{array}{l}\text { ¿Se publica la relación de puestos de trabajo de la Corporación y las } \\
\text { retribuciones del personal laboral y funcionario del ayuntamiento, } \\
\text { según las categorías? }\end{array}$ & 3 & 6,7 & 4 & 8,9 & 7 & 15,6 \\
\hline $\begin{array}{l}\text { ¿Se publica el listado y las retribuciones de los directivos y de los } \\
\text { cargos de confianza, sus funciones y su currículum? }\end{array}$ & 8 & 17,8 & 6 & 13,3 & 11 & 24,4 \\
\hline $\begin{array}{l}\text { ¿Se publica la oferta de trabajo del ayuntamiento, el desarrollo de los } \\
\text { concursos públicos y los resultados? }\end{array}$ & - & - & 33 & 73,3 & 34 & 75,6 \\
\hline ¿Se publica el inventario general del patrimonio del ayuntamiento? & - & - & 15 & 33,3 & 19 & 42,2 \\
\hline ¿Se publican todos los contratos formalizados, mayores y menores? & & - & 14 & 31,1 & 17 & 37,8 \\
\hline $\begin{array}{l}\text { ¿Se publican las licitaciones en curso y la composición de las mesas } \\
\text { de contratación? }\end{array}$ & - & - & 30 & 66,7 & 32 & 71,1 \\
\hline ¿Se publican las actas de las mesas de contratación? & - & - & 12 & 26,7 & 21 & 46,7 \\
\hline $\begin{array}{l}\text { ¿Se publican las modificaciones de los contratos formalizados y sus } \\
\text { prórrogas, las licitaciones anuladas y las resoluciones anticipadas? }\end{array}$ & - & - & 17 & 37,8 & 15 & 33,3 \\
\hline $\begin{array}{l}\text { ¿Se publica la relación completa de proveedores, adjudicatarios y/o } \\
\text { contratistas y la cuantía económica? }\end{array}$ & - & - & 3 & 6,7 & 7 & 15,6 \\
\hline ¿Se publica el periodo medio de pago a los proveedores? & - & - & 19 & 42,2 & 15 & 33,3 \\
\hline $\begin{array}{l}\text { ¿Se publican las subvenciones otorgadas, las respectivas convocatorias } \\
\text { y resoluciones? }\end{array}$ & - & - & 13 & 28,9 & 10 & 22,2 \\
\hline $\begin{array}{l}\text { ¿Se publican los convenios firmados, especificando las partes, su } \\
\text { objeto y las obligaciones económicas que se deriven, en su caso? }\end{array}$ & - & - & 8 & 17,8 & 17 & 37,8 \\
\hline $\begin{array}{l}\text { ¿Se publica el coste y las características de las campañas de publicidad } \\
\text { institucional en los medios de comunicación? }\end{array}$ & - & - & 3 & 6,7 & 6 & 13,3 \\
\hline
\end{tabular}

Fuente: Elaborada por los autores, 2019.

\section{Información para la Participación}

\section{Indicadores sobre qué información proporcionan sobre el municipio y la gestión de recursos colectivos}

Respecto a la Tabla 4, en primer lugar, es importante destacar que la mayoría de los ayuntamientos de la Región de Murcia hacen uso de la página web para publicar noticias, que a su vez suelen tratar temas sobre las actividades socioculturales del municipio o actuaciones de los miembros del gobierno. En raras ocasiones hacen referencia a la gestión como tal.

Más concretamente, la publicación de noticias relacionadas directamente con la actuación del gobierno y su gestión ha incrementado considerablemente a lo largo del tiempo, pasando de un 48\% en 2015 a más de un 80\% en 2016 y 2017. Por el contrario, respecto a las noticias relacionadas con las actuaciones de la oposición vemos que no ha habido ningún cambio en las tres oleadas. 
Tabla 4. Porcentaje de cumplimiento de los indicadores del nivel de transparencia sobre qué información proporcionan sobre el municipio y la gestión de recursos colectivos. Región de Murcia, España, 2019.

\begin{tabular}{|c|c|c|c|c|c|c|}
\hline \multirow{2}{*}{ Indicador } & \multicolumn{2}{|c|}{2015} & \multicolumn{2}{|c|}{2016} & \multicolumn{2}{|c|}{2017} \\
\hline & $n$ & $\%$ & $n$ & $\%$ & $n$ & $\%$ \\
\hline $\begin{array}{l}\text { ¿Se publican noticias, informaciones y/u opiniones sobre las actuaciones de los } \\
\text { miembros del gobierno relacionadas con la gestión del gobierno? }\end{array}$ & 22 & 48,9 & 38 & 84,4 & 39 & 86,7 \\
\hline $\begin{array}{l}\text { ¿Se publican noticias, informaciones y/u opiniones sobre las actuaciones de } \\
\text { los miembros de la oposición y/o de los grupos políticos relacionadas con el } \\
\text { control de la gestión del gobierno? }\end{array}$ & 2 & 4,4 & 2 & 4,4 & 2 & 4,4 \\
\hline $\begin{array}{l}\text { ¿Se publican noticias sobre el desarrollo del pleno, las mociones presentadas } \\
\text { por los diferentes grupos políticos, el debate y los acuerdos? }\end{array}$ & - & - & 11 & 24,4 & 6 & 13,3 \\
\hline ¿Se publica información histórica sobre el municipio? & 39 & 86,7 & 41 & 91,1 & 37 & 82,2 \\
\hline $\begin{array}{l}\text { ¿Se da información sobre la situación del municipio: datos sobre el término } \\
\text { municipal, la población empadronada y la diversidad social, actividades } \\
\text { económicas y culturales? }\end{array}$ & 22 & 48,9 & 32 & 71,1 & 27 & 60,0 \\
\hline ¿Se ofrece en la web una agenda de actividades municipales y ciudadanas? & 33 & 73,3 & 26 & 57,8 & 31 & 68,9 \\
\hline $\begin{array}{l}\text { ¿Se publica el contacto con la persona responsable de Prensa, Información y / } \\
\text { o Comunicación de la Corporación? }\end{array}$ & 8 & 17,8 & 16 & 35,6 & 15 & 33,3 \\
\hline
\end{tabular}

Fuente: Elaborada por los autores, 2019.

Cabe destacar la poca atención que se le presta a la publicación sobre el desarrollo de los plenos y las mociones, una información clave para el ciudadano que, sin embargo, no supera el 15,0\% en 2017 (disminuye el cumplimiento de este indicador respecto a 2016). En las tres oleadas detectamos que se dedica especial atención a publicar información de interés ciudadano sobre la historia del municipio, sobre todo en 2016 (91,1\%), aunque desciende ligeramente en 2017 (82,2\%).

Respecto a la información sobre los datos del responsable de prensa municipal, en ninguna de las tres oleadas encontramos que se haya cumplido el indicador. Hay un ligero incremento respecto a 2015. Este dato es de gran interés si tenemos en cuenta que, pese a la creciente profesionalización de la comunicación institucional con la que cuentan los ayuntamientos en la actualidad, no se alcanza el suficiente cumplimiento de este indicador.

Por otro lado, es importante destacar algunos de los indicadores básicos que no mejoran de una oleada a otra o, si lo hacen, no mantienen la tendencia de mejora. Tal es el caso de la publicación sobre la agenda cultural (que en 2015 alcanzaba un 73,0\%, en 2016 era de un 57,8\% y en 2017 de un 68,9\%.), así como la información sobre la localización y datos sociodemográficos del municipio (que ha pasado del 48,9\% al 71,1\% y después al 60,0\%).

Apenas se detectaron, en este caso, diferencias en función del sexo de los alcaldes, más allá del indicador relativo a la publicación de noticias, informaciones y opiniones sobre las actuaciones de los miembros del gobierno relacionadas con la gestión del gobierno. En este caso, como viene siendo habitual, los alcaldes obtienen mejor grado de cumplimiento $(71,1 \%)$ que las alcaldesas.

\section{Indicadores sobre qué herramientas ofrecen participación ciudadana}

Respecto a las herramientas de participación y control, los datos de la Tabla 5 muestran que son bastante deficientes en todos los Ayuntamientos de la Región de Murcia. En este sentido, aunque se pretende mejorar la participación ciudadana, se observa una situación deficitaria cuando hablamos de estas herramientas.

Es el caso de la información sobre los reglamentos o normas de participación ciudadana, aunque ha ido incrementando de una oleada a otra, los ayuntamientos no cumplen con dicho indicador. Lo mismo ocurre con la información de los mecanismos o entes de información, solo un 31\% de los ayuntamientos lo incluyen en 2017. 
Tabla 5. Porcentaje de cumplimiento de los indicadores del nivel de transparencia sobre qué información proporcionan sobre las herramientas que ofrecen para la participación ciudadana en el control democrático. Región de Murcia, España, 2019.

\begin{tabular}{|c|c|c|c|c|c|c|}
\hline \multirow{2}{*}{ Indicador } & \multicolumn{2}{|c|}{2015} & \multicolumn{2}{|c|}{2016} & \multicolumn{2}{|c|}{2017} \\
\hline & $\mathrm{n}$ & $\%$ & $n$ & $\%$ & $n$ & $\%$ \\
\hline $\begin{array}{l}\text { ¿Se da información en la web sobre el Reglamento de Participación Ciudadana } \\
\text { u otras normas al respecto? }\end{array}$ & 11 & 24,4 & 17 & 37,8 & 19 & 42,2 \\
\hline $\begin{array}{l}\text { ¿Se da información en la web de otros mecanismos o entes de participación: } \\
\text { consejos territoriales, consejos de ciudad, consejos sectoriales, etc.? }\end{array}$ & 4 & 8,9 & 5 & 11,1 & 14 & 31,1 \\
\hline $\begin{array}{l}\text { ¿Se publican las Actas de las reuniones de los otros mecanismos o entes de } \\
\text { participación mencionados en el indicador } 47 \text { ? }\end{array}$ & 1 & 2,2 & 5 & 11,1 & 7 & 15,6 \\
\hline $\begin{array}{l}\text { ¿Se ofrece en la web el directorio de entidades y asociaciones del municipio y/o } \\
\text { el registro de los grupos de interés, en su caso? }\end{array}$ & 23 & 51,1 & 22 & 48,9 & 16 & 35,6 \\
\hline $\begin{array}{l}\text { ¿Se ofrecen en la web instrumentos de consultas y/o de participación sobre } \\
\text { temas actuales de interés local? }\end{array}$ & & & 7 & 15,6 & 12 & 26,7 \\
\hline $\begin{array}{l}\text { ¿Se ofrece en la web la relación de servicios que se prestan (Carta de Servicios) } \\
\text { y los compromisos ante la ciudadanía? }\end{array}$ & - & & 8 & 17,8 & 3 & 6,7 \\
\hline $\begin{array}{l}\text { ¿Se proporcionan en la web instrumentos para valorar los servicios y para } \\
\text { presentar quejas o sugerencias sobre su funcionamiento? }\end{array}$ & 36 & 80,0 & 30 & 66,7 & 32 & 71,1 \\
\hline
\end{tabular}

Fuente: Elaborada por los autores, 2019.

Por otro lado, es importante destacar que el porcentaje de cumplimiento de la información sobre el directorio de entidades y asociaciones del municipio, así como el registro de los grupos de interés ha ido descendiendo progresivamente (ha pasado de un 51,1\% en 2015 a un 35,6\% en 2017).

Asimismo, llama la atención algo tan básico como es el buzón municipal de quejas y sugerencias que, si bien estaba presente en el 80,0\% de los casos en 2016, posteriormente su frecuencia disminuyó al 66,7 en 2016 y al $71,1 \%$ en 2017).

Para el caso de la información que proporciona herramientas para la participación ciudadana en el control democrático se encontraron algunos indicadores que resultaron tener una relación significativa con la variable sexo del alcalde. Concretamente, en 2015, los alcaldes, considerablemente con mayor frecuencia que las alcaldesas, ofrecían información en la web municipal sobre la relación de servicios del ayuntamiento, así como los compromisos adquiridos con la ciudadanía, además de información sobre instrumentos para valorar dichos servicios y para presentar quejas o sugerencias sobre su funcionamiento. Por su parte, en 2016, también los ediles fueron más transparentes $(70,6 \%)$ a la hora de dar información en la web sobre el reglamento de participación ciudadana.

\section{Discusión}

Esta investigación ofrece una reflexión teórica que se completa con un caso de estudio sobre la importancia de la transparencia como bien intangible del sector público, con el fin de evaluar los aspectos de la transparencia que afectan a la difusión online de la información pública y al suministro de las herramientas de participación a nivel local.

Tras analizar empíricamente una serie de indicadores sobre los que se concreta el nivel de transparencia online ofrecido en las páginas web de los 45 municipios que componen la Región de Murcia, como caso de estudio, y haciendo un seguimiento durante tres oleadas consecutivas (2015, 2016 y 2017) podemos concluir que las páginas web de los ayuntamientos de la Región de Murcia presentan insuficiencias respecto a los indicadores que hemos empleado para medir la transparencia.

Este dato corrobora los resultados recogidos en la literatura existente sobre el uso de la transparencia en los contextos locales, ya que, en líneas generales, los ayuntamientos de la Región de Murcia no utilizan la transparencia 
para ayudar a la gestión de la intangibilidad de la administración pública, con lo que esto dificulta la recuperación de la confianza y la credibilidad de los ciudadanos. En este sentido, la transparencia en aquellas cuestiones de gran interés para restablecer esa confianza de los ciudadanos, tales como los indicadores que abordan la publicación de retribuciones mensuales y anuales de los alcaldes y alcaldesas o que tratan sobre la información de sus declaraciones de bienes y actividades, sigue causando temor a los representantes políticos locales. Por otro lado, tampoco fomenta el aumento de credibilidad el hecho de que no se publique la agenda del alcalde y la alcaldesa, lo que impide que los ciudadanos puedan hacer un seguimiento de las funciones que desempeñan sus representantes políticos. Como resultado, nos encontramos ante organizaciones públicas que no responden a la demanda de ofrecer un gobierno más abierto que pueda garantizar la participación ciudadana.

Más concretamente, nuestro estudio demuestra que la hipótesis uno, en la que se reconoce que "existen diferencias significativas en lo que se refiere al cumplimiento de los indicadores de transparencia en función del tiempo transcurrido" no se cumple. De tal forma que, conforme pasa el tiempo, "el grado de cumplimiento de todos los indicadores es mayor" (confirmar el comienzo de las comillas) no se cumple en el caso de todos los indicadores. Sí se cumple con relación a la publicación de noticias relacionadas directamente con la actuación del gobierno y su gestión, cuyo porcentaje aumenta a lo largo del tiempo, lo cual fomenta la confianza por parte de los ciudadanos. Sin embargo, cuando hablamos de la información básica sobre los representantes políticos municipales, los datos muestran que solo hay mejora en la información sobre la biografía y el currículum. Y, respecto a la oposición, el cumplimiento de los indicadores es bastante menor a pesar de que aumenta de un año a otro. Tampoco se cumple cuando se habla de información sobre el responsable de prensa municipal.

En cuanto a la hipótesis dos, referida a si existen diferencias significativas en lo que se refiere al cumplimiento de los indicadores de transparencia en función del sexo del alcalde del municipio, este estudio ha demostrado que sí se cumple, puesto que se han detectado diferencias significativas en el grado de cumplimiento. Sin embargo, la mayor presencia de mujeres alcaldesas en la Región de Murcia no ha significado una mejora en los niveles de transparencia sino todo lo contrario: los mejores resultados se han encontrado cuando son los hombres los que están al frente de los gobiernos locales.

En definitiva, a pesar de que los gobiernos locales se vieron obligados a adaptarse y cumplir con la Ley 19/2013 (España, 2013) antes de finales de diciembre de 2015, los datos de nuestra investigación revelan que, desde 2015 a 2017, existen indicadores que siguen manteniendo un bajo grado de cumplimiento, que en ningún caso cumplen los mínimos establecidos legalmente. Dicho con otros términos, los ayuntamientos no se han sentido obligados a cumplir con la ley debido, en parte, a la inexistencia de mecanismos de control para hacer exigibles estos criterios. Esta falta de sensibilidad hacia el cumplimiento de los requisitos legales que afectan a la transparencia informativa se evidenció, incluso, entre un buen número de ayuntamientos que, tras ofrecerles asesoramiento gratuito para conocer sus resultados obtenidos, no consideraron necesaria la ayuda que se les ofreció para que pudieran actualizar y mejorar los mecanismos de información pública.

Sin duda, es necesario que las administraciones locales sean conscientes de la importancia de realizar una buena gestión de sus bienes intangibles. La percepción de la organización pública mejorará si todos los ciudadanos pueden acceder y comprender la información para que pueda existir bidireccionalidad. Si esta gestión no se realiza de una forma óptima no se puede dar respuesta a las exigencias sociales de transparencia y excelencia en el servicio público ofrecido por los gobiernos locales.

\section{Colaboradores}

CM ROBLES ha contribuido en la revisión de la literatura, en el diseño metodológico, en la redacción y análisis de los resultados y en las conclusiones. R ZAMORA-MEDINA ha contribuído en la revisión de la literatura, en el diseño metodológico del estudio, en la explotación final de los datos y sus correspondientes análisis estadísticos. 


\section{Referencias}

Alcaraz-Quiles, F. J.; Navarro-Galera, A.; Ortiz-Rodríguez, D. La transparencia sobre sostenibilidad en gobiernos regionales: el caso de España. Convergencia Revista de Ciencias Sociales, n. 73, p. 113-140, 2017. Disponible en: http://www.scielo.org. mx/pdf/conver/v24n73/1405-1435-conver-24-73-00113-en. pdf. Acceso en: 11 marzo 2020.

Alguacil-Gómez, J. Los desafíos del nuevo poder local: ¿hacia una estrategia relacional y participativa en el gobierno de la ciudad?. In: Alguacil-Gómez, J. (ed.). Poder local y participación democrática. Barcelona: El Viejo Topo, 2006. p. 9-47.

Alonso-Muñoz, A.; Casero-Ripollés, A. Transparencia y monitorización en el entorno digital. Hacia una tipología de las plataformas impulsadas por la ciudadanía. Revista Latina de Comunicación Social, v. 72, p. 1.351-1.366, 2017. Disponible en: http://repositori.uji.es/xmlui/bitstream/ handle/10234/172530/57180.pdf?sequence=1. Acceso en: 11 marzo 2020.

Bauhr, M.; Grimes, M. Indignation or resignation: the implications of transparency for societal accountability. Governance, v. 27, n. 2, p. 291-320, 2014.

Beltrán-Orenes, P.; Martínez Pastor, E. Grado de cumplimiento de las leyes de transparencia, acceso y buen gobierno, y de reutilización de los datos de contratación de la Administración central Española. El profesional de la información, v. 25, n. 4, p. 557-567, 2016. Disponible en: http://eprints.rclis. org/30087/1/557-567_Beltran_Martinez.pdf Acceso en: 11 marzo 2020.

Bossi, A.; Fuentes, Y.; Serrano, C. Reflexiones en torno a la aplicación del capital intelectual en el sector público. Revista Española de Financiación y Contabilidad, v. 34 n. 124, p. 211-245, 2005.

Cabezuelo-Lorenzo, F.; Rey-García, P.; Tapia-Frade, A. Análisis de las herramientas de control ciudadano sobre los representantes públicos: la transparencia informativa municipal en Castilla y León. Revista Latina de Comunicación Social, n. 71, p. 1.261-1.279, 2016.

Canel, M. J. La investigación sobre los bienes intangibles en la administración pública. En: Canel, M. J.; Piqueiras, P.; Ortega, G. (ed.). La comunicación de la Administración Pública: conceptos y casos prácticos de bienes intangibles. Madrid: Innap Investiga, 2017a. p. 11-28.

Canel, M. J. Qué aportan los bienes intangibles a la Administración Pública? En: Canel, M. J.; Piqueiras, P.; Ortega, G. (ed.). La comunicación de la Administración Pública: conceptos y casos prácticos de bienes intangibles. Madrid: Innap Investiga, 2017b. p. 29-50.

Canel, M. J. La Comunicación de la Administración Pública: para gobernar con la sociedad. México, D.F: Fondo de Cultura Económica, 2018.

Cinca, C.; Molinero, C.; Bossi, A. The measurement of intangible assets in public sector using scaling techniques. Journal of Intellectual Capital, v. 4, n. 2, p. 249-275, 2003. Available from: https://eprints.soton.ac.uk/35730/1/AF01-4.pdf. Access: 11 Mar. 2020.

España. Ministerio de la Presidencia Relaciones com las Cortes y Memoria Democratica. Ley 19/2013, de 9 de diciembre, de transparencia, acceso a la información pública y buen gobierno. Boletín Oficial del Estado: seccion 1, Madrid, n. 295, p. 97922- 97952, 10 dic. 2013.

García, J. Gobierno abierto: transparencia, participación y colaboración en las Administraciones Públicas. Innovar: Revista de Ciencias Administrativas y Sociales, v. 24, n. 54, p. 75-88, 2014.

Gértrudix, M.; Gertrudis-Casado, MC.; Álvarez-García, S. "Consumpton of public insttutons' open data by Spanish citzens". El profesional de la información, v. 25, n. 4, p. 535-544, 2016.

Guerrero, E. Responsabilidad y control: rendición de cuentas del Gobierno. In: Martínez, A. (ed.). Representación y calidad de la democracia en España. Madrid: Tecnos, 2006. p. 165-178.

Han, B. C. La sociedad de la transparencia. Barcelona: Herder Editorial, 2013.

International Business Machines Corporation. SPSS Statistics for Windows. Version 21.0. New York: IBM, 2012.

Kim, S.; Lee, J. E-participation, transparency, and trust in local government. Public Administration Review, v. 72, n. 6, p. 819828, 2012. Available from: http://faculty.cbpp.uaa.alaska.edu/ afgjp/PADM601\%20Fall\%202012/E\%20participation\%20 and\%20Trust\%20in\%20Local\%20Govt.pdf. Access: Mar. 11 2020.

Labio Bernal, A. Comunicación, periodismo y control informativo. Barcelona: Anthropos, 2006.

López-López, P. C., Vaca-Tapia, A. C., Rodríguez-Navas, P. La transparencia en las televisiones del Ecuador: una revisión legal de la información pública. Revista ComHumanitas, v. 8 n. 1, p. 137-158, 2017.

Luoma-aho, V. L.; Makikangas, M. E. Do public sector mergers (re)shape reputation? International Journal of Public Sector Management, v. 27, n. 1, p. 39-52, 2014.

MacNamara, J. Competence, competencies and/or capabilities for public communication? A public sector study. Asia Pacific Public Relations Journal, v. 19, p. 16-40, 2018.

Manfredi-Sánchez, J. L. et al. Transparencia y rendición de cuentas de los municipios de Castilla-La-Mancha. Barataria, n. 21, p. 175-189, 2016.

Manfredi-Sánchez, J. L. Horizontes de la información pública. El Profesional de la Información, v. 26, n. 3, p. 353-360, 2017. Disponible en: https://recyt.fecyt.es/index.php/EPI/article/ view/epi.2017.may.01 Acceso en: 11 marzo 2020.

Molina-Rodríguez-Navas, P. Ciberperiodismo e interactividad: entre la participación y la apariencia. Anàlisi Quaderns de Comunicació i Cultura, v. 36, p. 175-182, 2008.

Moreno-Sardá, A. et al. Infoparticip@: periodismo para la participación ciudadana en el control democrático: criterios, metodologías y herramientas. Estudios sobre el Mensaje Periodístico, v. 19, n. 2, p. 783803, 2013. Doi: http://dx.doi. org/10.5209/rev_esmp.2013.v19.n2.43471.

Moreno-Sardà, A.; Molina-Rodríguez-Navas, P.; Simelio-Solà, N. Impacto de la legislación sobre transparencia en la 
información publicada por las administraciones locales. El Profesional de la Información, v. 26, n. 3, p. 370-380, 2017. Doi: http://dx.doi.org/10.3145/epi.2017.may.03.

Pasquier, M.; Villeneuve, J. P. Marketing management and communications in the public sector. 2nd. ed. London: Routledge, 2017. Doi: http://dx.doi.org/10.4324/9781315622 309.

Poveda, J. E. G.; Tituaña, S. P. P.; Franco, P. Y. V. Importancia del control interno en el sector público. Revista Publicando, v. 3, n. 8, p. 487-502, 2016.

Queiroz, A. B.; Callén, Y. F.; Cinca, C. S. Reflexiones en torno a la aplicación del capital intelectual en el sector público. Revista Española de Financiación y Contabilidad, v. 34, n. 124, p. 211-245, 2005.

Rebolledo, M.; Zamora-Medina, R.; Rodríguez-Virgili, J. Transparency, accountability and participation in the local governments: comparative studies among Spanish Council's websites. Media studies, v. 7, n. 14, p. 48-64, 2016.

Rebolledo, M.; Zamora-Medina, R.; Rodríguez-Virgili, J. Transparency in citizen participation tools and public information: a comparative study of the Spanish city councils' websites. El profesional de la información, v. 26, n. 3, p. 361-369, 2017. Disponible en: https://dadun.unav.edu/ bitstream/10171/51690/1/pdf.pdf. Acceso en: 11 marzo 2020

Reig, R. Bases teóricas y documentales para el estudio de la Estructura de la información y el análisis estructural de los mensajes. Estudios sobre el Mensaje Periodístico, n. 15, p. 385-407, 2009.

Robles, C. M. La reputación y la legitimidad como bienes intangibles en el sector público: el caso del Ministro y el Ministerio de Educación, Cultura y Deporte (2011-2015). Tesis (Doctoral). Universidad Complutense de Madrid, Madrid, 2017. Disponible en: https://eprints.ucm.es/44275/1/T39126. pdf Acceso en: 11 marzo 2020.

Robles, C. M.; Rodríguez, C. El bien intangible legitimidad. En: Canel, M. J.; Piqueiras, P.; Ortega, G. (ed.). La comunicación de la Administración Pública: conceptos y casos prácticos de bienes intangibles. Madrid: Innap Investiga, 2017. p. 51-74.
Rubiños, M. Transparencia y valor intangible en el sector público. In: Canel, M. J.; Piqueiras, P.; Ortega, G. (ed.). La comunicación de la Administración Pública: conceptos y casos prácticos de bienes intangibles. Madrid: Innap Investiga, 2017. p. 177-188.

Schiavi, P. Aportes para una nueva agenda en la lucha contra la corrupción: buena administración, control social, transparencia, capacitación y comunicación. Estudios de Derecho Administrativo, n. 15, p. 281-314, 2017.

Stasavage, D. Transparency, democratic accountability and the economic consequences of monetary institutions. American Journal of Political Science, v. 47, n. 3, p. 389-402, 2003. Available fron: http://eprints.lse.ac.uk/186/1/Transparency. pdf. Access: 11 Mar. 2020.

Stirton, L.; Lodge, M. Transparency mechanisms: building publicness into public services. Journal of Law and Society, v. 28, n. 4, p. 471-489, 2001.

Transparency International España. Guía de linguaje claro sobre lucha contra la corrupción. Madrid: Transparency International España, 2009. Disponible en https:// transparencia.org.es/wp-content/uploads/2014/10/ Gu\%C3\%ADa-de-lenguaje-claro-sobre-lucha-contra-lacorrupci\%C3\%B3n.pdf. Acceso em: 7 abr. 2020

Uvalle-Berrones, R. Fundamentos de la transparencia en la sociedad contemporánea. Revista Mexicana de Ciencias Políticas y Sociales, v. 61, n. 226, p. 199-220, 2016. Disponible en: https://www.sciencedirect.com/science/article/pii/ S0185191816300083. Acceso en: 11 marzo 2020

Verge, T. Modelos alternativos de participación ciudadana en los partidos políticos españoles: un estudio del PSOE, el PP e IU. Revista Española de Ciencia Política, n. 17, p. 155-177, 2007.

Villoria-Mendieta, M. El largo camino hacia la transparencia en los Ayuntamientos españoles. Revista Técnica Especializada en Administración Local y Justicia Municipal, n. 18, p. 1983-2001, 2015.

Zamora-Medina, R. (ed). Claves para gestionar la comunicación política local. Salamanca: Editorial Comunicación Social, 2011. 